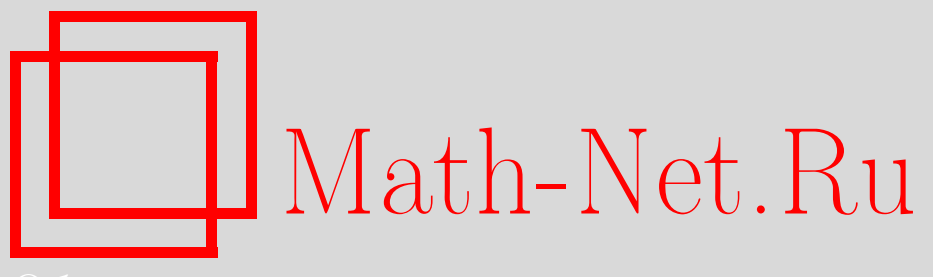

В. М. Круглов, Нормальная и пуассоновская сходимости, Теория вероятн. и ее примен., 2003, том 48, выпуск 2, 392-398

DOI: https://doi.org/10.4213/tvp292

Использование Общероссийского математического портала Math-Net.Ru подразумевает, что вы прочитали и согласны с пользовательским соглашением

http://www . mathnet.ru/rus/agreement

Параметры загрузки:

IP : 54.198 .187 .58

26 апреля 2023 г., 10:49:35

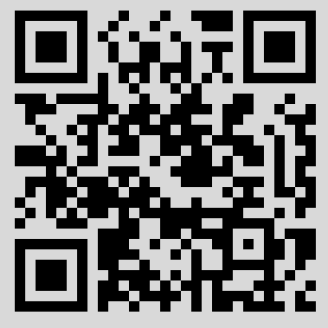


(C) 2003 г.

КРУГЛОВ В. М.*

\title{
НОРМАЛЬНАЯ И ПУАССОНОВСКАЯ СХОДИМОСТИ ${ }^{1)}$
}

\begin{abstract}
Приведены необходимые и достаточные условия для слабой сходимости распределений сумм независимых случайных величин к нормальному и пуассоновскому распределениям в терминах моментов не выше четвертого порядка урезанных слагаемых и некоторого условия, гарантируюшего асимптотическое равенство распределений упомянутых сумм и сумм урезанных слагаемых. Доказанные теоремы представляют собой аналоги известных критериев нормальной и пуассоновской сходимостей.

Ключевые слова и фразы: нормальная сходимость, пуассоновская сходимость, теорема Линдеберга-Феллера, слабая сходимость, моменты случайных величин.
\end{abstract}

Предельные теоремы для сумм независимых случайных величин излагались с разной степенью подробности во многих монографиях и учебниках с тех пор, как они были впервые изложены в монографии [1]. Из большого числа теорем неизменно выделяют теоремы о сходимости распределений сумм к нормальному, пуассоновскому и вырожденному распределениям. О причинах особого интереса к этим трем теоремам можно узнать, в частности, иг введения упомянутой монографии [1]. Формулировки этих трех теорем переписываются авторами новых монографий в том виде, в каком они появились в [1]. В предлагаемой заметке теоремам о сходимости к нормальному и пуассоновскому распределениям придается другая форма. Эти новые формулировки базируются на характеризациях нормального и пуассоновского распределений в классе безгранично делимых распределений. У нас нет полной уверенности в том, что характеризации этих распределений, особенно нормального, являются новыми. С другой стороны, в доступной нам литературе найти их не удалось.

1. Характеризации нормального и пуассоновского распределений. Пусть $\mathbf{N}$ - множество натуральных чисел, $\mathbf{R}-$ множество вещественных чисел, $\Phi$ стандартная нормальная функция распределения, $\Pi_{\lambda}(x)=\sum_{k<x} \lambda^{k} e^{-\lambda} / k !, x \in \mathbf{R}$, $k \in \mathbf{N},-$ пуассоновская функция распределения с параметром $\lambda>0$.

Условимся говорить, что функция распределения $F$ имеет нормальный тип, если $F(\sigma x+a)=\Phi(x)$ для всех $x \in \mathbf{R}$ и для некоторых $a \in \mathbf{R}$ и $\sigma>0$. Функция распределения $F$ имеет пуассоновский тип, если $F(x+a)=\Pi_{\lambda}(x)$ для всех $x \in \mathbf{R}$ или $F(x+a)=1-\Pi_{\lambda}(-x+)$ для всех $x \in \mathbf{R}$ и для некоторых $a \in \mathbf{R}$ и $\lambda>0$. Мы пользуемся стандартным обозначением $\Pi_{\lambda}(-x+)$ для предела справа указанной функции в точке $-x$.

Пусть $F-$ некоторая функция распределения. Первый момент (математическое ожидание) $\alpha$ и центральный момент $k$-го порядка $\beta_{k}$ функции распределения $F$ вычисляются по известным формулам $\alpha=\int_{-\infty}^{\infty} x d F(x), \beta_{k}=\int_{-\infty}^{\infty}(x-\alpha)^{k} d F(x), k \in \mathbf{N}$. Если $F(\sigma x+a)=\Phi(x)$, то стандартные вычисления приводят к следующим равенствам: $\alpha=a, \beta_{2 k+1}=0, \beta_{2 k}=\sigma^{2 k}(2 k) ! /\left(k ! 2^{k}\right), k \in \mathbf{N}$. Если $F(x+a)=\Pi_{\lambda}(x)$, то $\alpha=a+\lambda, \beta_{2}=\beta_{3}=\lambda, \beta_{4}=\lambda+3 \lambda^{2}$. Если $F(x+a)=1-\Pi_{\lambda}(-x+)$, то $\alpha=a-\lambda$, $\beta_{2}=\lambda, \beta_{3}=-\lambda, \beta_{4}=\lambda+3 \lambda^{2}$.

Теорема 1. Пусть $F$ - невырожденная безгранично делимая функция распределения с конечным абсолютным моментом четвертого порядка, $\alpha$ - математическое ожидание $u \beta_{k}, k=2,3,4$, - чентральные моменты $F$.

* Московский государственный университет им. М. В. Ломоносова, факультет вычислительной математики и кибернетики, Ленинские горы, 119992 Москва, Россия; e-mail: vkrug@dataforce.net

1) Работа выполнена при поддержке Российского фонда фундаментальных исследований, гранты 02-01-01080, 02-01-00949, 01-01-00644. 
(i) Для того чтобы функиия распределения $F$ имела нормальный тип, необходимо и достаточно, чтобы $\beta_{4} \leqslant 3 \beta_{2}^{2}$.

(ii) Для того чтобы Функиия распределения $F$ имела пуассоновский тип, необходимо и достаточно, чтобы $\beta_{2} \leqslant\left|\beta_{3}\right| u \beta_{4} \leqslant \beta_{2}+3 \beta_{2}^{2}$. Более того, если дополнительно известно, что $\alpha=\beta_{2} \leqslant \beta_{3}$, то $F$ совпадает $c \Pi_{\lambda}$.

Д о к а з а т е л ь с т в о. Докажем сначала (i). Если функция распределения $F$ имеет нормальный тип, то $\beta_{4}=3 \beta_{2}^{2}$. Пусть $F$ - невырожденная безгранично делимая функция распределения с конечным абсолютным моментом четвертого порядка и выполнено неравенство $\beta_{4} \leqslant 3 \beta_{2}^{2}$. Характеристическая функция, соответствующая $F$, может быть записана по формуле Колмогорова (см. $[1$, с. 92])

$$
f(t)=\exp \left\{i \gamma t+\int_{-\infty}^{\infty}\left(e^{i t x}-1-i t x\right) x^{-2} d K(x)\right\}, \quad t \in \mathbf{R},
$$

где $\gamma$ - вещественное число, $K$ - неубывающая, непрерывная слева, ограниченная функция, определенная на $\mathbf{R}$. Указанное представление $f$ единственно, если $K(-\infty)=0$. Мы будем предполагать, что последнее условие выполнено. Воспользовавшись известным равенством $f^{(k)}(0)=i^{k} \int_{-\infty}^{\infty} x^{k} d F(x)$ между $k$-м моментом функции распределения $F$ и $k$-й производной характеристической функции $f$, вычисленной в нуле, мы получим

$$
\beta_{2}=\int_{-\infty}^{\infty} d K(x), \quad \beta_{3}=\int_{-\infty}^{\infty} x d K(x), \quad \beta_{4}=3\left(\int_{-\infty}^{\infty} d K(x)\right)^{2}+\int_{-\infty}^{\infty} x^{2} d K(x) .
$$

Отсюда и из предполагаемого неравенства $\beta_{4} \leqslant 3 \beta_{2}^{2}$ следует, что $K(x)=0$, если $x \leqslant 0$, и $K(x)=\sigma^{2}>0$ - постоянная, если $x>0$. Обращаясь к формуле (1), мы видим, что функция распределения $F$ имеет нормальный тип.

Докажем теперь (ii). Если функция распределения $F$ имеет пуассоновский тип, то $\beta_{2}=\left|\beta_{3}\right|$ и $\beta_{4}=\beta_{2}+3 \beta_{2}^{2}$. Пусть $F$ - невырожденная безгранично делимая функция распределения с конечным абсолютным моментом четвертого порядка и выполняются неравенства $\beta_{2} \leqslant\left|\beta_{3}\right|$ и $\beta_{4} \leqslant \beta_{2}+3 \beta_{2}^{2}$. Из (2) и из неравенства $\beta_{4} \leqslant \beta_{2}+3 \beta_{2}^{2}$ следует, что $\int_{-\infty}^{\infty} x^{2} d K(x) \leqslant \beta_{2}$. Последнее неравенство вместе с предположением, что $\beta_{2} \leqslant\left|\beta_{3}\right|$, дают

$$
\int_{-\infty}^{\infty} x^{2} d K(x) \int_{-\infty}^{\infty} d K(x) \leqslant\left|\beta_{3}\right|^{2}=\left(\int_{-\infty}^{\infty} x d K(x)\right)^{2} .
$$

По неравенству Коши-Буняковского правая часть неравенства (3) не превосходит левой части, и, следовательно, оно обращается в равенство. Неравенство КошиБуняковского (3) обрашается в равенство тогда и только тогда, когда сушествует вешественное число $b$ такое, что $\int_{-\infty}^{\infty}(x-b)^{2} d K(x)=0$. Последнее равенство означает, что $K(x)=0$, если $x \leqslant b$, и $K(x)=\lambda>0$ - постоянная, если $x>b$. Привлекая (2), мы получим, что $\beta_{2}=\lambda, \beta_{3}=b \lambda, \beta_{4}=3 \lambda^{2}+b^{2} \lambda$. Отсюда и из предполагаемых неравенств $\beta_{2} \leqslant\left|\beta_{3}\right|$ и $\beta_{4} \leqslant 3 \beta_{2}^{2}+\beta_{2}$ вытекает, что $|b|=1$. Характеристическая функция (1) принимает вид $f(t)=\exp \left\{i t(\gamma-\lambda b)+\lambda\left(e^{i t b}-1\right)\right\}$, и, следовательно, $F$ имеет пуассоновский тип. Далее, если дополнительно известно, что $\alpha=\beta_{2} \leqslant \beta_{3}$, то $b=1$, $\gamma=\lambda$ и, следовательно, $F=\Pi_{\lambda}$. Теорема доказана.

2. Аналоги классических критериев. Пусть на вероятностном пространстве $(\Omega, \mathscr{F}, \mathbf{P})$ определены случайные величины $X_{n, k}, k=1, \ldots, m_{n}, n \in \mathbf{N}$, независимые для каждого $n \in \mathbf{N}$ и удовлетворяющие условию предельной равномерной малости:

$$
\lim _{n \rightarrow \infty} \max _{1 \leqslant k \leqslant m_{n}} \mathbf{P}\left\{\left|X_{n, k}\right|>\varepsilon\right\}=0, \quad \forall \varepsilon>0 .
$$

Пусть $\tau$ - фиксированное положительное число, $I[A]-$ индикаторная функция множества $A \subseteq \Omega, S_{n}=X_{n, 1}+\cdots+X_{n, m_{n}}, X_{n, k}(\tau)=X_{n, k} I\left[\left|X_{n, k}\right| \leqslant \tau\right], S_{n}(\tau)=$ $X_{n, 1}(\tau)+\cdots+X_{n, m_{n}}(\tau), F_{n}-$ функция распределения случайной величины $S_{n}(\tau)$. В формулировках нижеследующих теорем будут фигурировать математические ожидания и центральные моменты второго - четвертого порядков сумм $S_{n}(\tau)$. Подчеркнем, что предлагаемые условия для нормальной и пуассоновской сходимостей, на 
самом деле, выражаются в терминах отдельных слагаемых, так как величины $\mathbf{E} S_{n}(\tau)$, $\mathbf{E}\left[S_{n}(\tau)-\mathbf{E} S_{n}(\tau)\right]^{2}$ и $\mathbf{E}\left[S_{n}(\tau)-\mathbf{E} S_{n}(\tau)\right]^{3}$ являются суммами аналогичных моментов слагаемых и

$$
\begin{aligned}
\mathbf{E}\left[X_{n, k}(\tau)-\mathbf{E} X_{n, k}(\tau)\right]^{4}= & \sum_{k=1}^{m_{n}} \mathbf{E}\left[X_{n, k}(\tau)-\mathbf{E} X_{n, k}(\tau)\right]^{4} \\
& +6 \sum_{1 \leqslant k<l \leqslant m_{n}} \mathbf{E}\left[X_{n, k}(\tau)-\mathbf{E} X_{n, k}(\tau)\right]^{2} \mathbf{E}\left[X_{n, l}(\tau)-\mathbf{E} X_{n, l}(\tau)\right]^{2}
\end{aligned}
$$

Следующая теорема представляет собой аналог известного (см. $[1$, с. 136] или [2, c. 329]) критерия нормальной сходимости. Можно надеяться, что предлагаемые условия в некоторых случаях окажутся более удобными для проверки.

Теорема 2. Пусть даны числа $\alpha \in \mathbf{R} u \sigma>0$. Предположим, что выполнено условие (4). Для того чтобы

$$
\lim _{n \rightarrow \infty} \mathbf{P}\left\{S_{n}<x\right\}=\Phi\left(\sigma^{-1}(x-\alpha)\right), \quad \forall x \in \mathbf{R},
$$

необходимо и достаточно, чтобы для некоторого $\tau>0$

$$
\begin{gathered}
\lim _{n \rightarrow \infty} \sum_{k=1}^{m_{n}} \mathbf{P}\left\{\left|X_{n, k}\right|>\tau\right\}=0, \quad \lim _{n \rightarrow \infty} \mathbf{E} S_{n}(\tau)=\alpha, \\
\lim _{n \rightarrow \infty} \mathbf{E}\left[S_{n}(\tau)-\mathbf{E} S_{n}(\tau)\right]^{2}=\sigma^{2}, \quad \lim _{n \rightarrow \infty} \mathbf{E}\left[S_{n}(\tau)-\mathbf{E} S_{n}(\tau)\right]^{4} \leqslant 3 \sigma^{4} .
\end{gathered}
$$

Д оказ а те льст в о. Н е о 6 х о д и м о с т. Если выполнены (4) и (5), то условия (6) и первое условие в (7) выполняются по упомянутому выше критерию нормальной сходимости. Привлекая неравенства

$$
\mathbf{P}\left\{\left|S_{n}-S_{n}(\tau)\right|>0\right\} \leqslant \mathbf{P}\left\{\max _{1 \leqslant k \leqslant m_{n}}\left|X_{n, k}\right|>\tau\right\} \leqslant \sum_{k=1}^{m_{n}} \mathbf{P}\left\{\left|X_{n, k}\right|>\tau\right\}
$$

и первое условие в (6), мы видим, что последовательность $\left\{F_{n}\right\}_{n \geqslant 1}$ слабо сходится к нормальной функции распределения с параметрами $\alpha$ и $\sigma^{2}$. Убедимся, что последовательность случайных величин $\left\{\left|S_{n}(\tau)-\mathbf{E} S_{n}(\tau)\right|^{4}\right\}_{n \geqslant 1}$ равномерно интегрируема. Для этого достаточно доказать, что

$$
\sup _{n \in \mathbf{N}} \mathbf{E}\left|S_{n}(\tau)-\mathbf{E} S_{n}(\tau)\right|^{6}<\infty
$$

С целью доказать (9) воспользуемся равенством

$$
\begin{aligned}
& \mathbf{E}\left|S_{n}(\tau)-\mathbf{E} S_{n}(\tau)\right|^{6}=\sum_{k=1}^{m_{n}} \mathbf{E}\left|X_{n, k}(\tau)-\mathbf{E} X_{n, k}(\tau)\right|^{6} \\
& \quad+15 \sum_{1 \leqslant i \neq j \leqslant m_{n}} \mathbf{E}\left|X_{n, i}(\tau)-\mathbf{E} X_{n, i}(\tau)\right|^{4} \mathbf{E}\left|X_{n, j}(\tau)-\mathbf{E} X_{n, j}(\tau)\right|^{2} \\
& \quad+20 \sum_{1 \leqslant i<j \leqslant m_{n}} \mathbf{E}\left[X_{n, i}(\tau)-\mathbf{E} X_{n, i}(\tau)\right]^{3} \mathbf{E}\left[X_{n, j}(\tau)-\mathbf{E} X_{n, j}(\tau)\right]^{3}+90 \sum_{1 \leqslant i<j<k \leqslant m_{n}} A_{i, j, k},
\end{aligned}
$$

где $A_{i, j, k}=\mathbf{E}\left|X_{n, i}(\tau)-\mathbf{E} X_{n, i}(\tau)\right|^{2} \mathbf{E}\left|X_{n, j}(\tau)-\mathbf{E} X_{n, j}(\tau)\right|^{2} \mathbf{E}\left|X_{n, k}(\tau)-\mathbf{E} X_{n, k}(\tau)\right|^{2}$. Так как $\left|X_{n, i}(\tau)-\mathbf{E} X_{n, i}(\tau)\right| \leqslant 2 \tau$, то

$$
\begin{aligned}
& \mathbf{E}\left|S_{n}(\tau)-\mathbf{E} S_{n}(\tau)\right|^{6} \leqslant(2 \tau)^{4} \sum_{k=1}^{m_{n}} \mathbf{E}\left|X_{n, k}(\tau)-\mathbf{E} X_{n, k}(\tau)\right|^{2} \\
& \quad+35(2 \tau)^{2}\left(\sum_{k=1}^{m_{n}} \mathbf{E}\left|X_{n, k}(\tau)-\mathbf{E} X_{n, k}(\tau)\right|^{2}\right)^{2}+90\left(\sum_{k=1}^{m_{n}} \mathbf{E}\left|X_{n, k}(\tau)-\mathbf{E} X_{n, k}(\tau)\right|^{2}\right)^{3} .
\end{aligned}
$$

Отсюда и из первого условия в (7) следует (9). По известной теореме (см. [2, с. 196])

$$
\lim _{n \rightarrow \infty} \mathbf{E}\left[S_{n}(\tau)-\mathbf{E} S_{n}(\tau)\right]^{4}=\int_{-\infty}^{\infty}(x-\alpha)^{4} d \Phi\left(\frac{x-\alpha}{\sigma}\right)=3 \sigma^{4} .
$$

Доказательство необходимости условий теоремы завершено. 
Д о с т а т о ч н о с т ь. Для любого $\varepsilon>0$ выполняется неравенство

$$
\mathbf{P}\left\{\left|X_{n, k}(\tau)\right|>\varepsilon\right\} \leqslant \mathbf{P}\left\{\left|X_{n, k}\right|>\varepsilon\right\}+\mathbf{P}\left\{\left|X_{n, k}\right|>\tau\right\} .
$$

Отсюда и из условия (4) следует, что слагаемые сумм $S_{n}(\tau), n \in \mathbf{N}$, удовлетворяют условию предельной равномерной малости. Из второго условия в (6) и из первого условия в $(7)$ вытекает, что последовательность функций распределения $\left\{F_{n}\right\}_{n} \geqslant 1$ условно слабо компактна. Пусть $\left\{F_{k_{n}}\right\}_{n \geqslant 1}$ - подпоследовательность последовательности $\left\{F_{n}\right\}_{n \geqslant 1}$ - слабо сходится к функции распределения $F$. По теореме Хинчина (см. $[1$, c. 123]) $F$ - безгранично делимая функция распределения. Обратим внимание, что снова выполняется (9). Привлекая второе условие в (6), условия (7) и упомянутую выше теорему (см. [2, с. 196), мы получим

$$
\begin{gathered}
\sigma^{2}=\lim _{n \rightarrow \infty} \mathbf{E}\left|S_{k_{n}}(\tau)-\mathbf{E} S_{k_{n}}(\tau)\right|^{2}=\int_{-\infty}^{\infty}(x-\alpha)^{2} d F(x), \\
3 \sigma^{4} \geqslant \lim _{n \rightarrow \infty} \mathbf{E}\left|S_{k_{n}}(\tau)-\mathbf{E} S_{k_{n}}(\tau)\right|^{4}=\int_{-\infty}^{\infty}(x-\alpha)^{4} d F(x) .
\end{gathered}
$$

По теореме 1(i) $F$ - нормальная функция распределения с математическим ожиданием $\alpha$ и дисперсией $\sigma^{2}$. Мы доказали, что каждая слабо сходящаяся подпоследовательность последовательности $\left\{F_{n}\right\}_{n} \geqslant 1$ имеет своим пределом нормальную функцию распределения с параметрами $\alpha$ и $\sigma^{2}$. Последнее означает, что сама последовательность $\left\{F_{n}\right\}_{n \geqslant 1}$ слабо сходится к нормальной функции распределения с указанными параметрами. Привлекая первое условие в (6) и неравенства (8), мы придем к (5). Теорема доказана.

Следуюшей нашей целью является придать новую форму знаменитой теореме Линдеберга-Феллера.

Теорема 3. Пусть дано число $\sigma>0$. Предположим, ито выполнено условие (4) $u$

$$
\sum_{k=1}^{m_{n}} \mathbf{E} X_{n, k}^{2}=\sigma^{2}, \quad \mathbf{E} X_{n, k}=0, \quad k=1, \ldots, m_{n}, \quad n \in \mathbf{N} .
$$

Для того чтобы

$$
\lim _{n \rightarrow \infty} \mathbf{P}\left\{S_{n}<x\right\}=\Phi\left(\frac{x}{\sigma}\right), \quad \forall x \in \mathbf{R}
$$

необходимо и достаточно, чтобы для некоторого $\tau>0$

$$
\lim _{n \rightarrow \infty} \sum_{k=1}^{m_{n}} \int_{|x|>\tau} x^{2} d \mathbf{P}\left\{X_{n, k}<x\right\}=0, \quad \lim _{n \rightarrow \infty} \mathbf{E}\left[S_{n}(\tau)-\mathbf{E} S_{n}(\tau)\right]^{4} \leqslant 3 \sigma^{4} .
$$

Д о к а з а т е л ь с т в о. Если выполнены (4), (10) и (11), то первое условие в (12) выполняется по теореме Линдеберга-Феллера (см. [1, с. 110] или [2, с. 294]). Второе условие в (12) вытекает из (9) и (11). Доказательство достаточности можно скопировать с незначительными изменениями с доказательства достаточности условий из предыдущей теоремы. Теорема доказана.

Заметим, что в условиях теоремы 3 второе условие в (12) равносильно условию $\lim _{n \rightarrow \infty} \mathbf{E} S_{n}^{4}(\tau) \leqslant 3 \sigma^{4}$. Для этого достаточно убедиться, что $\lim _{n \rightarrow \infty} \mathbf{E} S_{n}(\tau)=0$, так как $\mathbf{E}\left[S_{n}(\tau)-\mathbf{E} S_{n}(\tau)\right]^{4}=\sum_{k=0}^{4} C_{4}^{k} \mathbf{E} S_{n}^{k}(\tau)\left(\mathbf{E} S_{n}(\tau)\right)^{4-k}$ и справедливо (9). Требуемое утверждение является следствием условий (10), первого условия в (12) и следующих соотношений:

$$
\left|\mathbf{E} S_{n}(\tau)\right|=\left|-\sum_{k=1}^{m_{n}} \int_{|x|>\tau} x d \mathbf{P}\left\{X_{n, k}<x\right\}\right| \leqslant \frac{1}{\tau} \sum_{k=1}^{m_{n}} \int_{|x|>\tau} x^{2} d \mathbf{P}\left\{X_{n, k}<x\right\} .
$$

Классический критерий пуассоновской сходимости (см. [1, с. 141] или $[2$, с. 330]) также можно сформулировать в новой форме. 
Теорема 4. Пусть дано число $\lambda>0$. Предположим, что выполнено условие (4). Для того чтобы

$$
\lim _{n \rightarrow \infty} \mathbf{P}\left\{S_{n}<x\right\}=\Pi_{\lambda}(x)
$$

для любого $x \in \mathbf{R}$ - точки непрерьвности функиии $\Pi_{\lambda}$, необходимо и достаточно, чтобы для некоторого $\tau>1$

$$
\begin{aligned}
& \lim _{n \rightarrow \infty} \sum_{k=1}^{m_{n}} \mathbf{P}\left\{\left|X_{n, k}\right|>\tau\right\}=0, \quad \lim _{n \rightarrow \infty} \mathbf{E} S_{n}(\tau)=\lambda, \\
& \lim _{n \rightarrow \infty} \mathbf{E}\left[S_{n}(\tau)-\mathbf{E} S_{n}(\tau)\right]^{2}=\lambda \leqslant \lim _{n \rightarrow \infty} \mathbf{E}\left[S_{n}(\tau)-\mathbf{E} S_{n}(\tau)\right]^{3}, \\
& \lim _{n \rightarrow \infty} \mathbf{E}\left[S_{n}(\tau)-\mathbf{E} S_{n}(\tau)\right]^{4} \leqslant \lambda+3 \lambda^{2} .
\end{aligned}
$$

Д оказательство. Н е об ходи м о ть. Если выполнены условия (4) и (13), то условия (14) и первое условие в (15) выполняется по упомянутому выше критерию пуассоновской сходимости. Второе условие в (15) и условие (16) вытекают из (9), (13) и из теоремы 1 (ii).

Д о с т а т о ч н о с т ь. Из второго условия в (14) и первого условия в (15) следует, что последовательность $\left\{F_{n}\right\}_{n \geqslant 1}$ условно слабо компактна. Пусть $F-$ функция распределения, предельная для некоторой подпоследовательности последовательности $\left\{F_{n}\right\}_{n \geqslant 1}$. Рассуждая, как при доказательстве достаточности условий в теореме 2 , мы убедимся, что слагаемые сумм $S_{n}(\tau)$ удовлетворяют условию предельной равномерной малости. По теореме Хинчина (см. [1, с. 123]) $F$ - безгранично делимая функция распределения. Далее мы можем рассуждать, как рассуждали при доказательстве достаточности условий в теореме 2. Привлекая (9) и условия (14)-(16), мы убедимся в справедливости следующих соотношений:

$$
\int_{-\infty}^{\infty} x d F(x)=\lambda, \quad \int_{-\infty}^{\infty}(x-\lambda)^{2} d F(x)=\lambda, \quad \int_{-\infty}^{\infty}(x-\lambda)^{4} d F(x) \leqslant 3 \lambda^{2}+\lambda .
$$

По теореме 1 (ii) $F=\Pi_{\lambda}$. Мы доказали, что каждая слабо сходящаяся подпоследовательность последовательности $\left\{F_{n}\right\}_{n \geqslant 1}$ имеет своим пределом пуассоновскую функцию распределения $\Pi_{\lambda}$. Последнее означает, что сама последовательность $\left\{F_{n}\right\}_{n} \geqslant 1$ слабо сходится к $\Pi_{\lambda}$. Привлекая (8) и первое условие в (14), мы придем к (13). Теорема доказана.

3. Новая форма теоремы Золотарева. Современное состояние теории предельных теорем подытожено в монографии [3]. Одним из центральных результатов этой теории является теорема Золотарева, обобшаюшая теорему Линдеберга-Феллера на случай, когда условие (4) нарушено. В этом пункте мы приводим новый вариант теоремы Золотарева.

Предположим, что случайные величины $X_{n, k}, k=1, \ldots, m_{n}, n \in \mathbf{N}$, удовлетворяют условиям (10). Пусть $F_{n, k}$ - функция распределекия случайной величины $X_{n, k}$, $\Phi_{n, k}$ - нормальная функция распределения с нулевым математическим ожиданием и дисперсией $\mathbf{E} X_{n, k}^{2}, L$ - расстояние Леви (метрика на множестве функций распределения, определенных на вещественной прямой; ее определение приведено в $[1$, с. 38]), $\alpha_{n}=\max _{1 \leqslant k \leqslant m_{n}} L\left(F_{n, k}, \Phi_{n, k}\right), U_{n}=\left\{k \in\left\{1, \ldots, m_{n}\right\}: \mathbf{E} X_{n, k}^{2}<\sqrt{\alpha_{n}}\right\}$,

$B_{n, 2}(\tau)=\mathbf{E}\left[\sum_{k \in U_{n}}\left(X_{n, k}(\tau)-\mathbf{E} X_{n, k}(\tau)\right)\right]^{2}, \quad B_{n, 4}(\tau)=\mathbf{E}\left[\sum_{k \in U_{n}}\left(X_{n, k}(\tau)-\mathbf{E} X_{n, k}(\tau)\right)\right]^{4}$.

Величины $B_{n, 2}(\tau)$ и $B_{n, 4}(\tau)$ полагаются равными нулю, если $U_{n}$ - пустое множество.

Теорема 5. Пусть дано число $\sigma>0$. Предположим, что выполнены условия (10). Для того чтобы выполнялось (11), необходимо и достаточно, чтобы $\lim _{n \rightarrow \infty} \alpha_{n}=0$ и для некоторого $\tau>0$

$$
\lim _{n \rightarrow \infty} \sum_{k \in U_{n}} \int_{|x|>\tau} x^{2} d F_{n, k}(x)=0, \quad \liminf _{n \rightarrow \infty}\left(3 B_{n, 2}^{2}(\tau)-B_{n, 4}(\tau)\right) \geqslant 0 .
$$


Док а $з$ а т е льс т в о. Н е о 6 ход и м о ст ь. Если выполнены (10) и (11), то условие $\lim _{n \rightarrow \infty} \alpha_{n}=0$ и первое условие в (17) выполняются по теореме Золотарева (см. [3, с. 312] или [4]). Докажем второе условие в (17). Пусть $\bar{U}_{n}=$ $\left\{1, \ldots, m_{n}\right\} \backslash U_{n}, G_{n}^{1}, G_{n}^{2}, G_{n}^{3}$ - функции распределения соответственно $\sum_{k \in \bar{U}_{n}} X_{n, k}$ $\sum_{k \in U_{n}} X_{n, k}, \sum_{k \in U_{n}}\left(X_{n, k}(\tau)-\mathbf{E} X_{n, k}(\tau)\right)$. В силу условий (10) и неравенства Чебышева мы имеем, что $G_{n}^{k}(-x)+1-G_{n}^{k}(x) \leqslant x^{-2} \sigma^{2}, k=1,2$, для любого $x>0$. Последнее неравенство влечет условную слабую компактность последовательностей $\left\{G_{n}^{k}\right\}_{n \geqslant 1}$, $k=1,2$. Обратим внимание на следующие соотношения:

$$
\begin{gathered}
\mathbf{P}\left\{\left|\sum_{k \in U_{n}}\left(X_{n, k}-X_{n, k}(\tau)\right)\right|>0\right\} \leqslant \sum_{k \in U_{n}} \mathbf{P}\left\{\left|X_{n, k}\right|>\tau\right\} \leqslant \frac{1}{\tau^{2}} \sum_{k \in U_{n}} \int_{|x|>\tau} x^{2} d F_{n, k}(x), \\
\left|\sum_{k \in U_{n}} \mathbf{E} X_{n, k}(\tau)\right|=\left|-\sum_{k \in U_{n}} \int_{|x|>\tau} x d F_{n, k}\right| \leqslant \frac{1}{\tau} \sum_{k \in U_{n}} \int_{|x|>\tau} x^{2} d F_{n, k}(x) .
\end{gathered}
$$

Из этих соотношений и из первого условия в (17) следует, что последовательность $\left\{G_{n}^{3}\right\}_{n} \geqslant 1$ условно слабо компактна. Более того, предельные функции распределения для соответствующих слабо сходящихся подпоследовательностей последовательностей $\left\{G_{n}^{2}\right\}_{n \geqslant 1}$ и $\left\{G_{n}^{3}\right\}_{n \geqslant 1}$ совпадают. Пусть $\left\{k_{n}\right\}_{n \geqslant 1}$ - неубывающая последовательность натуральных чисел такая, что $\lim _{n \rightarrow \infty}\left(3 B_{k_{n}, 2}^{2}(\tau)-B_{k_{n}, 4}(\tau)\right)=$ $\liminf _{n \rightarrow \infty}\left(3 B_{n, 2}^{2}(\tau)-B_{n, 4}(\tau)\right)$. Выберем подпоследовательность последовательности $\left\{k_{n}\right\}_{n} \geqslant 1$ такую, чтобы соответствующие подпоследовательности последовательностей $\left\{G_{k_{n}}^{1}\right\}_{n \geqslant 1}$ и $\left\{G_{k_{n}}^{2}\right\}_{n \geqslant 1}$ слабо сходились. Без ограничения общности рассуждений можно считать, что сами эти последовательности слабо сходятся, скажем, к функциям распределения $G^{1}$ и $G^{2}$. В силу (11) свертка $G^{1}$ и $G^{2}$ является нормальной функцией распределения. По теореме Крамера (см. [2, с. 286]) $G^{1}$ и $G^{2}$ - нормальные функции распределения. Выше было отмечено, что последовательность $\left\{G_{k_{n}}^{3}\right\}_{n \geqslant 1}$ также слабо сходится к $G^{2}$. Рассуждая, как при доказательстве (9), мы убедимся, что $\sup _{n \in \mathbf{N}} \mathbf{E}\left|\sum_{k \in U_{n}}\left(X_{n k}(\tau)-\mathbf{E} X_{n, k}(\tau)\right)\right|^{6}<\infty$. По известной теореме (см. [2, с. 196])

$$
\begin{gathered}
0=\lim _{n \rightarrow \infty} \int_{-\infty}^{\infty} x d G_{k_{n}}^{3}(x)=\int_{-\infty}^{\infty} x d G^{2}(x), \\
\lim _{n \rightarrow \infty} B_{k_{n}, 2}=\int_{-\infty}^{\infty} x^{2} d G^{2}(x), \quad \lim _{n \rightarrow \infty} B_{k_{n}, 4}=\int_{-\infty}^{\infty} x^{4} d G^{2}(x) .
\end{gathered}
$$

Отсюда следует второе условие в (17), так как $G^{2}$ - нормальная функция распределения с нулевым математическим ожиданием.

Д о с т а т о ч н о с т ь. Из условий (10) следует, что последовательности $\left\{G_{n}^{1}\right\}_{n \geqslant 1}$ и $\left\{G_{n}^{2}\right\}_{n \geqslant 1}$ условно слабо компактны. Обозначим $\Phi_{n}^{1}$ свертку нормальных функций распределения $\Phi_{n, k}, k \in \bar{U}_{n}$, и $\sigma_{n, 1}^{2}=\sum_{k \in \bar{U}_{n}} \mathbf{E} X_{n, k}^{2}, \sigma_{n, 2}^{2}=$ $\sum_{k \in U_{n}} \mathbf{E} X_{n, k}^{2}$. В упомянутой статье [4] доказано, что равенство $\lim _{n \rightarrow \infty} L\left(G_{n}^{1}, \Phi_{n}^{1}\right)=0$ является следствием условия $\lim _{n \rightarrow \infty} \alpha_{n}=0$. Пусть $\left\{k_{n}\right\}_{n \geqslant 1}$ - возрастающая подпоследовательность натуральных чисел такая, что подпоследовательности $\left\{G_{k_{n}}^{1}\right\}_{n \geqslant 1}$ и $\left\{G_{k_{n}}^{2}\right\}_{n \geqslant 1}$ слабо сходятся, скажем, к функциям распределения $G^{1}$ и $G^{2}$, числовые последовательности $\left\{\sigma_{k_{n}, 1}^{2}\right\}_{n \geqslant 1}$ и $\left\{\sigma_{k_{n}, 2}^{2}\right\}_{n \geqslant 1}$ сходятся, скажем, к числам $\sigma_{1}^{2}$ и $\sigma_{2}^{2}$, числовые последовательности $\left\{B_{k_{n}, 2}\right\}_{n \geqslant 1}$ и $\left\{B_{k_{n}, 4}\right\}_{n \geqslant 1}$ сходятся, скажем, к числам $B_{2}$ и $B_{4}$. Если $\sigma_{2}^{2}=0$, то последовательности $\left\{\Phi_{k_{n}}^{1}\right\}_{n \geqslant 1}$ и $\left\{G_{k_{n}}^{2}\right\}_{n \geqslant 1}$ слабо сходятся соответственно к $\Phi(x / \sigma)$ и к функции распределения, вырожденной в нуле. Отсюда следует (11), так как последовательности $\left\{\Phi_{k_{n}}^{1}\right\}_{n \geqslant 1}$ и $\left\{G_{k_{n}}^{1}\right\}_{n \geqslant 1}$ имеют обший слабый предел. Предположим теперь, что $\sigma_{2}^{2}>0$. Слагаемые суммы $\sum_{k \in U_{n}} X_{n, k}$ удовлетворяют условию (4). По упомянутой теореме Хинчина (см. $\left[1\right.$, с. 123]) $G^{2}-$ безгранично делимая функция распределения. Как при доказательстве необходимости условий, мы убедимся, что последовательность $\left\{G_{n}^{3}\right\}_{n \geqslant 1}$ слабо компактна, предельные функции распределения для соответствующих слабо сходящихся подпоследовательностей последовательностей $\left\{G_{n}^{2}\right\}_{n \geqslant 1}$ и $\left\{G_{n}^{3}\right\}_{n \geqslant 1}$ совпадают и выполняются равенства (19) и $(20)$. В силу второго условия в (17) и теоремы $1(\mathrm{i}), G^{2}$ - нормальная функция распределения. Она имеет нулевое математическое ожидание и дисперсию, скажем, $\Delta_{2}^{2}$. 
Заметим, что $\sum_{k \in U_{n}}\left|\int_{-\infty}^{\infty} x^{2} d F_{n, k}(x)-\int_{|x| \leqslant \tau} x^{2} d F_{n, k}(x)\right| \leqslant \sum_{k \in U_{n}} \int_{|x|>\tau} x^{2} d F_{n, k}(x)$. Привлекая первое условие в (17), (18) и первое равенство в (20), мы убедимся, что $\Delta_{2}^{2}=\sigma_{2}^{2}$. Подытоживая, мы находим, что

$$
\lim _{n \rightarrow \infty} \mathbf{P}\left\{S_{k_{n}}<x\right\}=\lim _{n \rightarrow \infty} G_{k_{n}}^{1} * G_{k_{n}}^{2}(x)=\Phi\left(\sigma^{-1} x\right), \quad \forall x \in \mathbf{R} .
$$

Доказано, что любая слабо сходящаяся подпоследовательность последовательности распределений сумм $S_{n}$ имеет своим слабым пределом нормальную функцию распределения с нулевым математическим ожиданием и дисперсией $\sigma^{2}$. Это доказывает (11). Теорема доказана.

4. Заключительные замечания. В формулировках теорем 1-5 присутствуют условия, выраженные в виде неравенств между моментами случайных величин. На самом деле, эти неравенства обращаются в равенства. Тем не менее мы сформулировали эти условия в виде неравенств, считая, что в конкретных ситуациях доказать неравенства проще, чем доказать равенства.

Читатель, несомненно, заметил, что условие $\lim _{n \rightarrow \infty} \sum_{k=1}^{m_{n}} \mathbf{P}\left\{\left|X_{n, k}\right|>\tau\right\}=0$ заимствовано из традиционных формулировок критериев нормальной, пуассоновской и вырожденной сходимостей. В традиционных формулировках требуется, чтобы это условие выполнялось для любого $\tau>0$. В наших формулировках требуется, чтобы оно выполнялось для некоторого $\tau>0$.

Последнее наше замечание касается формулировки известного критерия вырожденной сходимости (см. $[1$, с. 143$]$ или $[2$, с. 331]). Нам представляется, что она страдает некоторой избыточностью. Сначала мы приведем нашу формулировку, а затем укажем на имеющуюся, по нашему мнению, избы'гочность в традиционной формулировке критерия.

Теорема 6. Пусть выполнено условие (4). Для того чтобы

$$
\lim _{n \rightarrow \infty} \mathbf{P}\left\{\left|S_{n}\right|>\varepsilon\right\}=0, \quad \forall \varepsilon>0,
$$

необходимо и достаточно, чтобы для некоторого $\tau>0$

$$
\begin{gathered}
\lim _{n \rightarrow \infty} \sum_{k=1}^{m_{n}} \mathbf{P}\left\{\left|X_{n, k}\right|>\tau\right\}=0, \\
\lim _{n \rightarrow \infty} \mathbf{E} S_{n}(\tau)=0, \quad \lim _{n \rightarrow \infty} \mathbf{E}\left|S_{n}(\tau)-\mathbf{E} S_{n}(\tau)\right|^{2}=0 .
\end{gathered}
$$

Д о к а $з$ а т е л ь с т в о. Если выполнены (4) и (21), то условия (22) и (23) выполняются по упомянутому выше критерию вырожденной сходимости.

Д о с т а т о ч н о с т ь. Если выполнены условия (23), то с помощью неравенства Чебышева легко убедиться, что последовательность сумм $\left\{S_{n}(\tau)\right\}_{n \geqslant 1}$ сходится по вероятности к нулю. Утверждение (21) теперь следует из (8) и (22). Теорема доказана.

Напомним, что традиционная формулировка критерия вырожденной сходимости гласит: утверждение (21) выполняется тогда и только тогда, когда условие (22) выполняется для любого $\tau>0$ и условия (23) выполняются для некоторого $\tau>0$. Требование, чтобы условие (22) выполнялось для любого $\tau>0$ страдает избыточностью.

\section{СПИСОК ЛИТЕРАТУРЫ}

1. Гнеденко Б. В., Колмогоров А. Н. Предельные распределения для сумм независимых случайных величин. М.: ГИТТЛ, 1949, 264 с.

2. Лоэв М. Теория вероятностей. М.: ИЛ, 1962, 719 с.

3. Золотарев В.M. Современная теория суммирования независимых случайных величин. М.: Наука, 1986, 415 с.

4. Золотарев B. М. Обобщение теоремы Линдеберга-Феллера. - Теория вероятн. и ее примен., 1967, т. 12 , в. 4 , с. $666-677$. 